\title{
Modelagem Matemática da Cinemática de um Braço Robótico Acionado Pneumaticamente
}

\author{
Claudio da Silva dos Santos, Sandra E. B. Viecelli, Antonio Carlos Valdiero, Luiz \\ Antonio Rasia
}

DECEEng/UNIJUÍ / Câmpus Panambi/ Caixa Postal 121/ CEP 98280-000, Panambi-RS, Brasil

E-mail: prof.claudiosant@hotmail.com; Sandra_edinara@hotmail.com; valdiero@unijui.edu.br; rasia@unijui.edu.br;

\begin{abstract}
Resumo: $O$ presente trabalho trata da modelagem matemática da cinemática direta de um braço robótico com dois graus de liberdades acionado por atuadores pneumáticos. O braço robótico possui diversas aplicações na indústria e em equipamentos agro-florestais e pode ser utilizado em atividades perigosas e insalubres como na pintura de peças industriais. O objetivo principal desse trabalho é deduzir as equações da cinemática direta utilizando a convenção de Denavit-Hartenberg, incluindo-se a definição dos parâmetros e a utilização destes no cálculo das matrizes de transformações homogêneas que relacionam os sistemas de referência dos elos, resultando no cálculo da localização exata da posição do efetuador final. Para fins de posicionamento do efetuador final, aplica-se um sinal de controle nas válvulas pneumáticas dos atuadores, resultando na movimentação do braço. A equação da cinemática direta de robôs permite o cálculo da posição e orientação do efetuador final (garra robótica ou ferramenta) a partir da medição dos ângulos de junta. Pretende-se assim, contribuir para o desenvolvimento de soluções robotizadas com acionamento pneumático para problemas da indústria e agro-industriais. Esta pesquisa contou com o apoio da CELPE no âmbito do Programa de P\&D da ANEEL, da FAPERGS e do CNPq.
\end{abstract}

\section{Introdução}

Este trabalho apresenta a modelagem matemática da cinemática de um braço robótico com dois graus de liberdade e considerando também as principais características não lineares.

Segundo [4], o uso de robôs industriais tem como objetivos principais tornar a produção automatizada a fim de reduzir custos dos produtos fabricados, aumentar a produtividade, utilizar melhor a matéria prima reduzindo perdas, economizar energia, melhorar as condições de trabalho fazendo atividades perigosas e insalubres e melhorar a qualidade do produto.

$\mathrm{O}$ uso de atuadores pneumáticos para o acionamento na robótica torna-se a alternativa viável, pois eles têm algumas vantagens como o baixo custo, leveza, durabilidade e são limpos, facilidade de manutenção, boa relação força/tamanho e flexibilidade de instalação e também a disponibilidade do ar comprimido na maioria das empresas [7]. Além disso, os atuadores pneumáticos também apresentam menor risco de contaminação ambiental e de operações em relação aos sistemas hidráulicos [3].

Em oposição a essas vantagens os sistemas pneumáticos possuem algumas características indesejáveis as quais dificultam as suas aplicações em respostas mais precisas. Estas características indesejáveis derivam da compressibilidade do ar e das não-linearidades presentes no sistema dentre as quais podemos destacar o atrito nas vedações do atuador.

De acordo com [2] no estudo da robótica, estamos constantemente interessados na localização de objetos no espaço tridimensional. Esses objetos são os elos do manipulador, as peças e ferramentas com as quais lida e outros objetos em seu ambiente. Em nível rudimentar, porém importante, tais objetos são descritos por apenas dois atributos: posição e orientação. 


\section{Descrição da bancada experimental do braço robótico pneumático}

O braço robótico utilizado para esse trabalho e do tipo SCARA com dois graus de liberdade e acionamento pneumático. Para o acionamento pneumático é utilizado dois atuadores pneumáticos de haste simples e dupla ação, ou seja, de avanço e recuo, sendo os mesmos responsáveis pelo movimento do braço robótico. $\mathrm{O}$ atuador 1 utilizado no movimento da junta um do braço possui curso de $200 \mathrm{~mm}$ e o atuador 2 responsável pelo deslocamento da junta dois possui curso de $160 \mathrm{~mm}$. As hastes estão conectadas ao êmbolo, que através do sinal de controle enviado por uma servoválvula permite posicionar o braço em um determinado curso dos cilindros ou seguir uma variável em função do tempo.

Os atuadores pneumáticos funcionam com o ar comprimido que é enviado pela servoválvula com certa pressão de suprimento $\left(p_{s}\right)$ devidamente ajustada.

Inicialmente, o sinal de controle $\mathrm{U}_{\mathrm{T}}$ energiza o solenoide da válvula de modo que uma força magnética resultante é aplicada no carretel da válvula, produzindo o deslocamento do carretel.

O deslocamento do mesmo abre o orifício de controle para que uma câmara do atuador linear seja ligada a linha de pressão de suprimento e a outra seja ligada à pressão atmosférica $\left(p_{a t m}\right)$.

Desta forma, uma diferença de pressão nas câmaras do atuador linear, dando origem a uma força resultante movendo o elo do braço robótico que está preso ao atuador, em deslocamento no sentido positivo ou negativo y, conectado diretamente ao sinal de entrada. A força produzida pelo atuador pneumático é dada pelo produto da área do êmbolo do atuador pela diferença de pressão nas câmaras e é chamada de força pneumática.

Para a captura das pressões são utilizados os sensores de pressão, que são os responsáveis por fazer a leitura das pressões iniciais nas câmaras a e b dos atuadores, assim como a pressão de suprimento manométrica (bar).

O sistema possui dois sensores elétricos que geram sinais mediante a rotação do eixo da junta, indicando assim a posição ou ângulo. A partir da rotação do eixo da junta, um disco perfurado gira, interrompendo o feixe de luz que chega até o sensor óptico. Este sensor é ligado a placa eletrônica dSPACE que converte o sinal do sensor na forma de pulsos para o valor correspondente ao deslocamento angular, ou seja a placa possui um contador de pulsos.

A bancada experimental de testes utilizada é composta por um microcomputador responsável pela visualização dos dados e interface com o operador. A este está interligado uma placa móvel dSPACE 1104 responsável pela captura e armazenagem dos dados da bancada de testes, a qual utiliza a integração dos softwares Matlab/Simulink e ControlDesk permitindo a captura, controle e manipulação dos dados em tempo real através da construção de uma interface gráfica, o que possibilita a análise detalhada dos resultados obtidos.

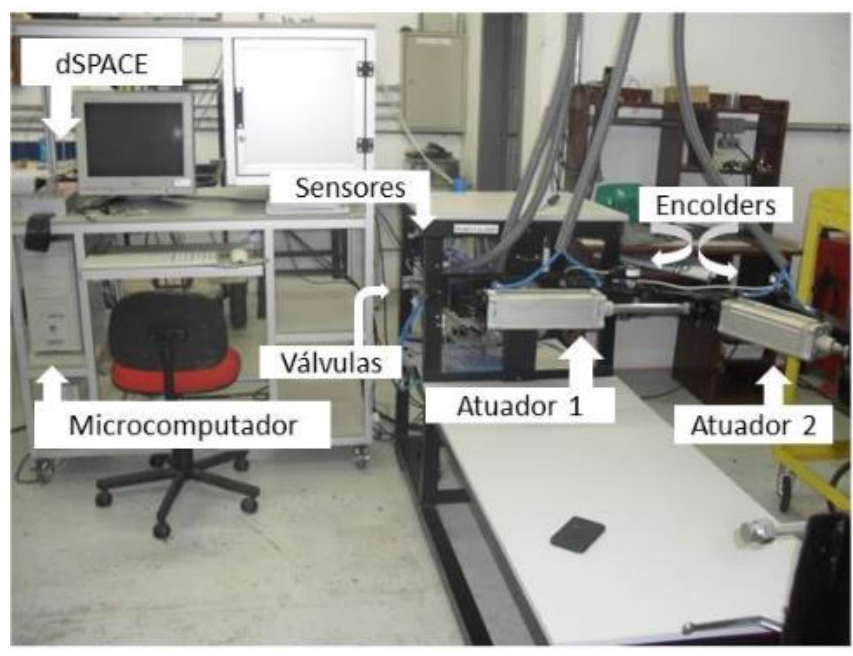

Figura 1: Fotografia da bancada do robô pneumático. 


\section{Modelagem Cinemática do braço robótico}

O sistema robótico depende sempre da posição e a orientação no espaço, sendo que estas estão atreladas a um sistemas de coordenadas ou sistema de referência.

Este sistema possibilita a localização de objetos no espaço tridimensional. Esses objetos pode ser considerado o próprio elo do robô, ou as peças e ferramentas que o mesmo possui.

Segundo [2], qualquer referencial pode servir como sistema de referência para expressar a posição e a orientação do corpo, de forma que quase sempre pensamos em transformar ou mudar a descrição desses atributos do corpo, de um sistema para outro.

A partir da definição de um sistemas de coordenadas podemos localizar qualquer ponto no universo através de um vetor de posição como mostra a equação 1 .

$$
{ }^{A} P=\left[\begin{array}{c}
P_{x} \\
P_{y} \\
P_{z}
\end{array}\right]
$$

onde ${ }^{A} P$ é um vetor e pode ser considerado uma posição no espaço, sendo que os dados subscritos x, y e z são os elementos individuais.

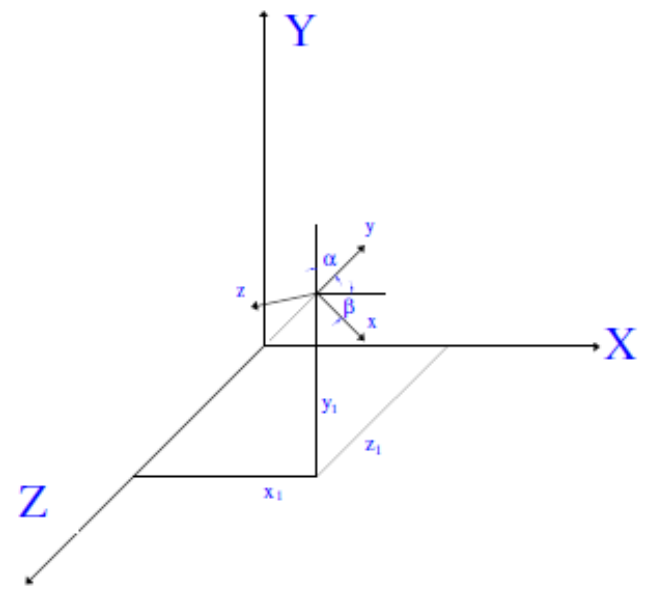

Figura 2. Vetor em relação ao sistema de referência Fonte:Valdiero(2005)

\section{Cinemática direta do braço robótico}

Neste trabalho utiliza-se a cinemática do braço robótico que permite posicionar e orientar o efetuador final do manipulador com os valores das variáveis de junta em um sistema de coordenadas fixo que descreve sua movimentação no tempo, não levando em consideração as forças que produzem este movimento.

O robô é controlado através de suas variáveis articulares, a realização do controle de posição em relação ao sistema de coordenadas cartesianas implica no desenvolvimento de metodologias para a transformação de coordenadas.

Para descrever a translação e rotação entre dois elos adjacentes utiliza-se um método matricial para o estabelecimento sistemático de um sistema de coordenadas fixo para cada elo da cadeia cinemática.

As relações cinemáticas entre os deslocamentos lineares das hastes dos atuadores e as rotações dos elos foram deduzidas conforme a metodologia de um modelo dinâmico de um manipulador serial com atuadores lineares e são definidas por parâmetros de localização do atuador em relação aos sistemas de referências da convenção de Denavit-Hartenberg [6]. 


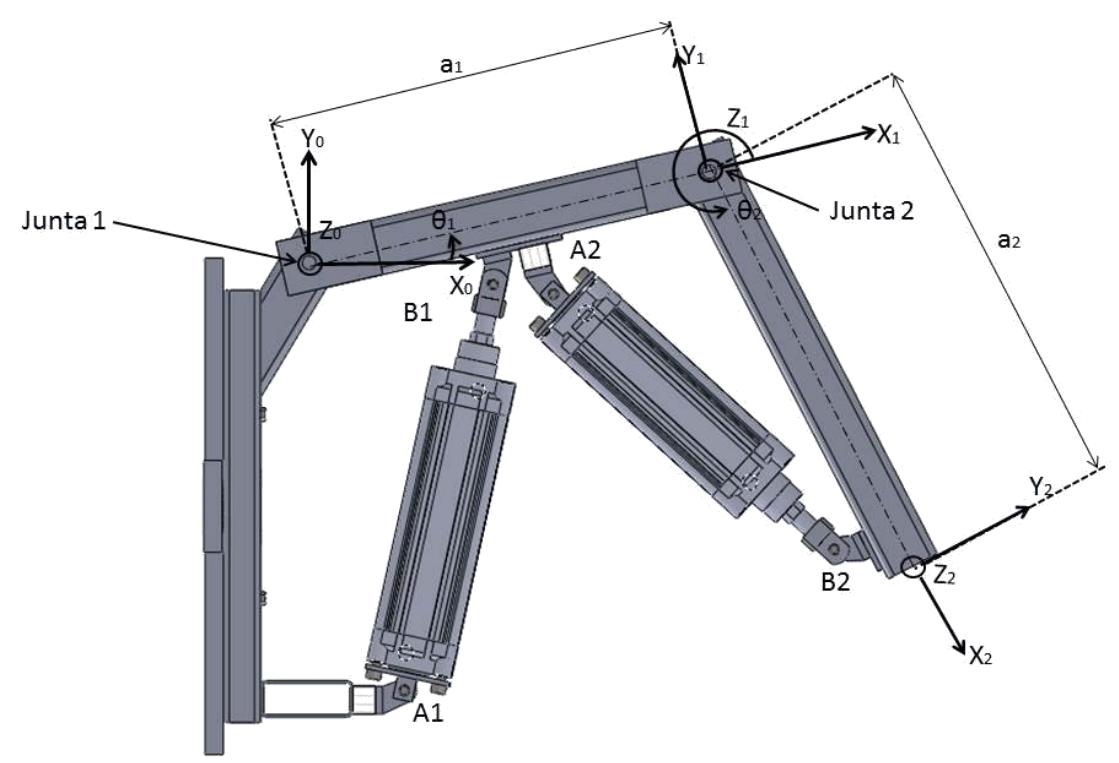

Figura 3.Vista lateral do mecanismo do manipulador robótico acionado pneumaticamente com indicação de parâmetros de Denavit-Hartenberg.

\section{Resultados}

A partir da definição do sistema de coordenadas de cada elo, a posição e orientação do sistema de referência i pode ser completamente especificados com relação ao sistema de referência i-1 que através da convenção de Denavit-Hartenberg estabelece os parâmetros como mostra a Tabela 1.

\begin{tabular}{ccccc}
\hline Elo i & $\theta_{i}(\mathrm{rad})$ & $d_{i}(m)$ & $a_{i}(m)$ & $\alpha_{i}(\mathrm{rad})$ \\
\hline 1 & $\theta_{1}$ & 0 & $a_{1}=0,455$ & 0 \\
2 & $\theta_{2}$ & 0 & $a_{2}=0,534$ & 0 \\
\hline
\end{tabular}

Tabela 1. Parâmetros de Denavit-Hertenberg

Uma vez determinados os parâmetros de Denavit- Hartenberg, é possível deduzir uma matriz de transformação homogênea que relaciona o sistema de referência(i) em relação ao sistema de relação (i-1).

$$
A_{i}^{i-1}\left(q_{i}\right)=\left[\begin{array}{cccc}
\cos \theta_{i} & -\operatorname{sen} \theta_{i} \cdot \cos \alpha_{i} & \operatorname{sen} \theta_{i} \cdot \operatorname{sen} \alpha_{i} & a_{i} \cdot \cos \theta_{i} \\
\operatorname{sen} \theta_{i} & \cos \theta_{i} \cdot \cos \alpha_{i} & -\cos \theta_{i} \cdot \operatorname{sen} \theta_{i} & a_{i} \cdot \operatorname{sen} \theta_{i} \\
0 & \operatorname{sen} \alpha_{i} & \cos \alpha_{i} & d_{i} \\
0 & 0 & 0 & 1
\end{array}\right]
$$


A partir os valores dos parâmetros de Denavit-Hartenberg faz-se o cálculo das matrizes de transformação homogênea conforme a Equação 2. Substituindo os parâmetros na matriz acima se calcula a matriz posição que transforma de 2 para 1, obtendo o seguinte resultado.

$$
A_{2}^{1}\left(q_{2}\right)=\left[\begin{array}{cccc}
\cos \theta_{2} & -\operatorname{sen} \theta_{2} & 0 & a_{2} \cdot \cos \theta_{2} \\
\operatorname{sen} \theta_{2} & \cos \theta_{2} & 0 & a_{2} \cdot \operatorname{sen} \theta_{2} \\
0 & 0 & 1 & 0 \\
0 & 0 & 0 & 1
\end{array}\right]
$$

De mesmo modo se calcula a matriz posição que transforma de 1 para 0.

$$
A_{1}^{0}\left(q_{1}\right)=\left[\begin{array}{cccc}
\cos \theta_{1} & -\operatorname{sen} \theta_{1} & 0 & a_{1} \cdot \cos \theta_{1} \\
\operatorname{sen} \theta_{1} & \cos \theta_{1} & 0 & a_{1} \cdot \operatorname{sen} \theta_{1} \\
0 & 0 & 1 & 0 \\
0 & 0 & 0 & 1
\end{array}\right]
$$

Utilizando cada uma das matrizes de transformação homogênea que relaciona o elo com o anterior, pode-se calcular a equação da Cinemática Direta por meio do produto da esquerda dado por:

$$
T_{2}^{0}\left(\theta_{1} \cdot \theta_{2}\right)=A_{1}^{0} \cdot A_{2}^{1}=\left[\begin{array}{cccc}
\vec{M} & \vec{S} & \vec{Q} & \vec{P} \\
0 & 0 & 0 & 1
\end{array}\right]
$$

Como resultados dessas multiplicações matriciais obtêm-se a posição $P_{x}$ dada por:

$$
P_{x}=a_{2} \cdot \cos \left(\theta_{1}+\theta_{2}\right)+a_{1} \cdot \cos \theta_{1}
$$

e também a posição $P_{y}$ dada por:

$$
P_{y}=a_{2} \cdot \operatorname{sen}\left(\theta_{1}+\theta_{2}\right)+a_{1} \cdot \operatorname{sen} \theta_{1}
$$

Calculada a matriz de transformação do sistema de referência do efetuador para o sistema da base, pode-se obter as relações da cinemática direta. Então, utilizando os valores dos parâmetros $a_{1}$ e $a_{2}$ dados $\theta_{1}$ e $\theta_{2}$, calculamos a posição $P$ do efetuador final dada por:

$$
P=\left[\begin{array}{c}
P_{x} \\
P_{y} \\
P_{z}
\end{array}\right]=\left[\begin{array}{c}
a_{2} \cos \left(\theta_{1}+\theta_{2}\right)+a_{1} \cos \theta_{1} \\
a_{2} \operatorname{sen}\left(\theta_{1}+\theta_{2}\right)+a_{1} \operatorname{sen} \theta_{1} \\
0
\end{array}\right]
$$

A equação7 descreve o planejamento de trajetória no espaço das juntas e constitui-se na relação cinemática entre a posição do efetuador final e os ângulos de das juntas. 


\section{Conclusão}

Este trabalho apresentou a modelagem matemática da cinemática de um manipulador robótico de dois graus de liberdade com acionamento pneumático, utilizando-se a convenção de Denavit-Hartenberg. As equações da cinemática direta são importantes para fins de planejamento de trajetória, simulação computacional e controle de posição do robô. Pretende-se utilizar os resultados numa aplicação industrial no Arranjo Produtivo Local Metal-Mecânico de cidade pólo em Panambi/RS.

\section{Agradecimentos}

$\mathrm{O}$ presente trabalho foi realizado com apoio do $\mathrm{CNPq}$, Conselho Nacional de Desenvolvimento Científico e Tecnológico - Brasil. Os autores também são agradecidos à Fundação de Amparo a Pesquisa do Rio Grande do Sul (FAPERGS) pelas bolsas de iniciação científica e desenvolvimento tecnológico, à UNIJUÍ pelo apoio e incentivo na realização da pesquisa, além da infraestrutura do Núcleo de Inovação em Máquina Automáticas e Servo Sistemas (NIMASS), implantado com recursos provenientes do SEBRAE e do FINEP/SEBRAE/MCT, num convênio de interação Universidades-Empresas do Arranjo Produtivo Local Metal-Mecânica, e modernizado recentemente com o apoio financeiro da CELPE (Companhia Energética de Pernambuco) por meio de um projeto de pesquisa aplicada no âmbito do Programa de P\&D da ANEEL.

\section{Referências}

[1] Bollmann, A., "Fundamentos da Automação Industrial Pneutrônica. Projeto de comandos Binários Eletropneumáticos", São Paulo, Associação Brasileira de Hidráulica e Pneumática,1997.

[2] Craing, J. J. , tradução Sousa, H.C. "Robótica", São Paulo , Person Education do Brasil,2012.

[3] M. R. Richer, "Modelagem Matemática e Controle de Posição de um Atuador Acionado Pneumaticamente", Dissertação de Mestrado, UNIJUÍ, 2013.

[4] Romano, V. F., ”Robótica Industrial”, São Paulo, Edgard Blucher LTDA,2002.

[5] Sciavicco, L.; Siciliano, B. ,'Modelig and control of robot manipulators. Naples: McGrawHill, 1996.

[6] Valdiero, A. C., "Modelagem Matemática de Robôs Hidraulicos”, Ijuí: Unijuí,2012.

[7] Wang, J.; Yang, L.; Luo, X.; Mangan, S.; Derby, J. W., "Mathematical Modeling Study of Scroll Air Motors and Energy Efficiency Analysis-Part I", (IEEE/ASME) pp 112-121 Trasaction on Mechatronics, 2011. 